\title{
Shoulder Pain Treated by Manual Acupuncture and Pharmacopuncture Following Origin/Insertion Technique of Applied Kinesiology: A Case Series of Two Patients
}

\author{
Chang-Beohm Ahn ${ }^{1}$, Joon-Yong Noh ${ }^{1}$, Hyun-Min Yoon ${ }^{2}$, Cheol-Hong Kim², Ung-Kwan Song ${ }^{3}$ \\ ${ }^{1}$ Department of Acupuncture \& Moxibustion Medicine, Samse Korean Medicine Hospital, Busan, Republic of Korea \\ ${ }^{2}$ Department of Acupuncture \& Moxibustion, College of Oriental Medicine, Dongeui University, Busan, Republic of Korea \\ ${ }^{3}$ Department of Family Medicine, Samse Medicine Hospital, Busan, Republic of Korea
}

Received October 10, 2021

Reviewed October 13, 2021

Accepted November 11, 2021

*Corresponding Author

Chang-Beohm Ahn

Department of Acupuncture \&

Moxibustion Medicine, Samse Korean

Meical Hospital, 1580 Jungang-daero,

Geumjeong-gu, Busan 46302, Republic of Korea

Tel: +82-51-580-6912

E-mail: cbahn@deu.ac.kr
The aim of this study was to report on the improvement of shoulder pain resulting from disorders of the rotator cuff such as impingement syndrome and adhesive capsulitis, by manual acupuncture (MA) and pharmacopuncture (PA) following origin/insertion technique (OIT) of applied kinesiology (AK). Two patients were treated with MA and PA after OIT on shoulder muscles. The Numerical Rating Scale and the assessment of the Japanese Orthopedic Association scores were used to assess the pain, and ultrasound images were taken to compare treatment outcome. This study showed that MA and PA following OIT may be an effective treatment for impingement syndrome and adhesive capsulitis.

Keywords: manual acupuncture, pharmacopuncture, origin/insertion technique of applied kinesiology, ultrasound imaging, japanese orthopedic association score

\section{INTRODUCTION}

Shoulder pain is due to the pathology of various sources such as neck, glenohumeraljoint, acromioclavicularjoint, rotator cuff, and other soft tissues around the shoulder joint. The most prevalent cause is disorders of the rotator cuff which include impingement syndrome and adhesive capsulitis [1].

Shoulder pain is diagnosed by radiological examination such as magnetic resonance imaging or ultrasound imaging, together with patient symptoms, and assessment of Japanese Orthopaedic Association score (JOAS) and Numerical Rating score (NRS) $[2,3]$.

Treatment of shoulder pain includes conservative therapies or surgery performed according to the degree of shoulder pain and radiological examination [4]. Acupuncture treatments include manual acupuncture (MA) and pharmacopuncture (PA) in Korean Medicine.

Origin/Insertion Technique (OIT) is a technique of strength- ening muscles by firm, heavy, digital stimulation on the origin and insertion of muscle $[5,6]$. Until recently, numerous clinical trials have been conducted investigating the effectiveness of MA and PA on shoulder pain [7-15]. However, few studies on shoulder pain have reported follow-up ultrasound changes after MA, PA, and OIT treatments. The objective of this case study was to report changes observed by JOA, NRS, and ultrasound images after treatments.

Informed consent for this case report was given by subjects for ethical approval.

\section{CASE REPORT}

\section{Treatment methods}

The whole process of diagnosis and treatment was done by Dr. Ahn who has over 50 years experience practicing Korean traditional medicine. 


\section{Diagnosis}

Cervical movements, flexion, extension, bilateral bending, and rotation were tested.

\section{Cervical-related muscles}

Muscles were tested such as levator scapulae, rhomboids, trapezium, and scalene medius.

\section{Shoulder joint movements}

Movements were tested such as abduction, adduction, elevation, internal rotation, and external rotation.

\section{Shoulder and scapularmuscles}

Muscles were tested such as supraspinatus, infraspinatus, subscapularis, teres minor, deltoid, as well as serratus anterior, and biceps brachii muscles.

\section{Other muscles}

Muscles were often tested such as subclavius, coracobrachialis, and pectoralis minor when pain was not improved by over 10 treatments.

\section{Origin/Insertion technique}

The OIT is applied to the sites (coracoids process and upper aspect of the greater tubercle of the humerus) of origin/insertion of the weak muscle by heavy digital massage with bilateral thumbs for 30-60 seconds to strengthen the weak muscle.

\section{Acupuncture interventions}

\section{1) Manual acupuncture}

The patients underwent 20 sessions of acupuncture (usually 3 to 5 sessions per week, for 5 to 7 weeks) and the point locations were chosen according to $\mathrm{WHO}$ acupuncture locations. The acupuncture needles were $40 \times 0.25 \mathrm{~mm}$. Fifteen acupoints (such as LU1, LU3, LU4, LU11, LI14, LI15, LI16, SI11, SI9, SI10, SI12, SI13, SI14, SI15, and ST38) were applied [16-18]. Patients were needled to a depth of approximately $1 \mathrm{~cm}$. Depending on symptoms, other points were also added such as GV16, BL10,
GB21, and TW14. The retention time was 20 minutes.

\section{2) Pharmacopuncture}

Pharmacopuncture is a treatment that combines acupuncture and herbal medicine. In this study, it was performed with disposable Kovax-syringes $(20 \mathrm{ml}, 23 \mathrm{G} \times 1$ 1": Korea Vaccine, Ansan, Korea) and $6 \mathrm{ccs}$ of refrigerated Sweet BV PA solution prepared by Jaseng Namyangju Industrial Institute (Namyangju, Korea), which is an extramural facility.

\section{3) Preparation of Sweet BV pharmacopuncture solution}

Sweet BV pharmacopuncture solution was needled at coracoids process, which is around the LU1 as well as the upper aspect of the greater tubercle of the humerus during MA.

\section{4) Evaluation}

\section{(1) Ultrasound imaging}

Taking ultrasound images of the rotating cuff muscle is the basic method to diagnose the causes of shoulder pain such as impingement of the cuff between the coracoacromial arch and humeral head as well as rotator cuff tears, and adhesive capsulitis. The images were taken before the $1^{\text {st }}$ time and after the $20^{\text {th }}$ time.

Numerical rating scale (NRS) is a scoring method to quantify the subjective severity of pain felt by the patient on a scale from 0 to 10 . The worst imaginable pain is scored as 10 and the pain-free state is scored as 0 [3]. Evaluation was done before the $1^{\text {st }}$ time and after the $20^{\text {th }}$ time.

(2) Japanese Orthopaedic Association score (JOAS)

The JOAS is generally used to assess the treatment result of shoulder conditions and includes evaluation of pain (30 points). Function (overall function: 10 points, activities of daily living: 10 points), range of motion (active motion: 30 points), radiologic findings (5 points), and joint stability (15 points), amounting to a full score of 100 points [2] (Table 1). Evaluation was done before the $1^{\text {st }}$ time and after the $20^{\text {th }}$ time.

\section{Case 1}

\section{1) Participant}

Kim XX (Female, 58 years old)

\section{2) Diagnosis by ultrasound}

The diagnoses were as follows:

(1) Mild impingement on the supraspinatus (SST) tendon. 
Chang-Beohm Ahn, et al.

Table 1. Assessing shoulder outcomes based on the Japanese Orthopaedic Association Score (JOAS)

\begin{tabular}{|c|c|c|c|}
\hline 1. Pain (30 points) & & 3. Range of motion (30 points) & \\
\hline None & 30 & Elevation & \\
\hline Tenderness or minimal pain during sports or heavy labor & 25 & $>150^{\circ}$ & 15 \\
\hline Minimal pain during activities of daily living & 20 & $>120^{\circ}$ & 12 \\
\hline Moderate and tolerance pain (occasional night pain) & 10 & $>90^{\circ}$ & 9 \\
\hline Severe pain (frequent night pain) & 5 & $>60^{\circ}$ & 6 \\
\hline Totally incapacitated because of pain & 0 & $>30^{\circ}$ & 3 \\
\hline 2. Function (20 points) & & $0^{\circ}$ & 0 \\
\hline Strength in abduction & & External rotation & \\
\hline Normal & 5 & $>60^{\circ}$ & 9 \\
\hline Excellent & 4 & $>30^{\circ}$ & 6 \\
\hline Good & 3 & $>0^{\circ}$ & 3 \\
\hline Fair & 2 & $>-20^{\circ}$ & 1 \\
\hline Poor & 1 & $<-20^{\circ}$ & 0 \\
\hline Zero & 0 & Internal rotation & \\
\hline Endurance (time in seconds of holding $1 \mathrm{~kg}$ ) & & Above the $\mathrm{T} 12$ spinous process & 6 \\
\hline$>10$ seconds & 5 & Above the $\mathrm{L} 5$ spinous process & 4 \\
\hline$>3$ seconds & 3 & Gluteal & 2 \\
\hline$<2$ seconds & 1 & Below gluteal & 0 \\
\hline 0 & 0 & 4. Radiographic evaluation (5 points) & \\
\hline Activities of daily living ( 1 point each) & & Normal & 5 \\
\hline Combing the hair & & Moderate changes & 3 \\
\hline Making knot in the back & & Advanced changes & 0 \\
\hline Reaching the mouth & & 5. Joint stability (15 points) & \\
\hline Reaching the opposite axilla & & Normal & 15 \\
\hline Reaching an overhead shelf & & Slight instability & 10 \\
\hline Reaching a side pocket (jacket) & & Severe instability & 5 \\
\hline Putting on a jacket & & Relevant history & 0 \\
\hline \multicolumn{4}{|l|}{ Opening and closing a sliding door } \\
\hline \multicolumn{4}{|l|}{ Self-hygienic care } \\
\hline Sleeping on the involved side & & Total & 100 \\
\hline
\end{tabular}

(2) Partial tear on the proximal SST (40-50\%).

\section{3) Chief complaint}

Right neck pain, shoulder pain, limitation of movement, and severe night pain.

\section{4) Onset and cause}

Onset was July 22 due to contusion of shoulder to road while walking.

\section{5) Present status}

Hospitalized at an orthopaedic clinic on July 22 for two weeks.

\section{6) Treatment period}

The patient was treated between Aug. 2021 and Sep. 2021.

7) Progress (Fig. 1, Table 2)

Progress in the treatment ( $\mathrm{tx})$ of case 1

Shoulder pain 2021.08.10 (before treatment): 2021.09.30 (after 20 treatments) 
A.

10

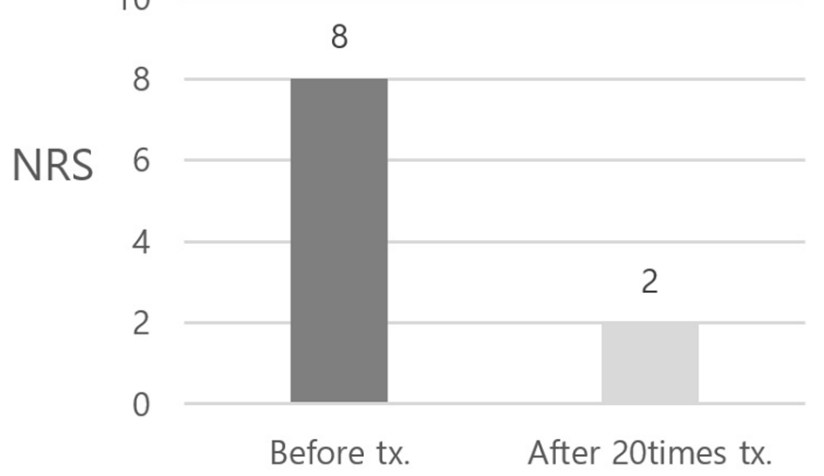

B.

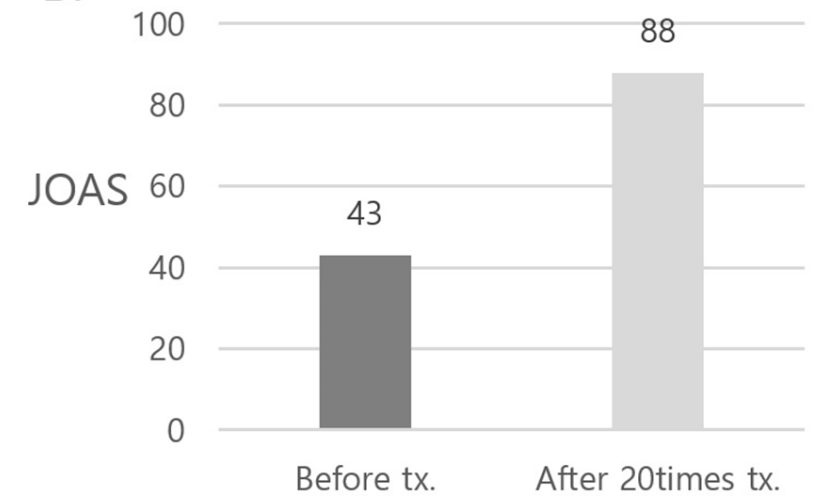

Figure 1. Assessment of pain in Case 1 (A: numerical rating score, NRS, B: Japanese Orthopaedic Association Score, JOAS).

Table 2. JOAS of Case I

\begin{tabular}{lcc}
\hline & Before tx. & After 20 times tx. \\
\hline Pain (30) & 5 & 20 \\
Function (20) & 6 & 20 \\
Range of motion (30) & 19 & 30 \\
Radiograph evaluation (5) & 3 & 3 \\
Joint stability (15) & 10 & 15 \\
Total (100) & 43 & 88 \\
\hline
\end{tabular}

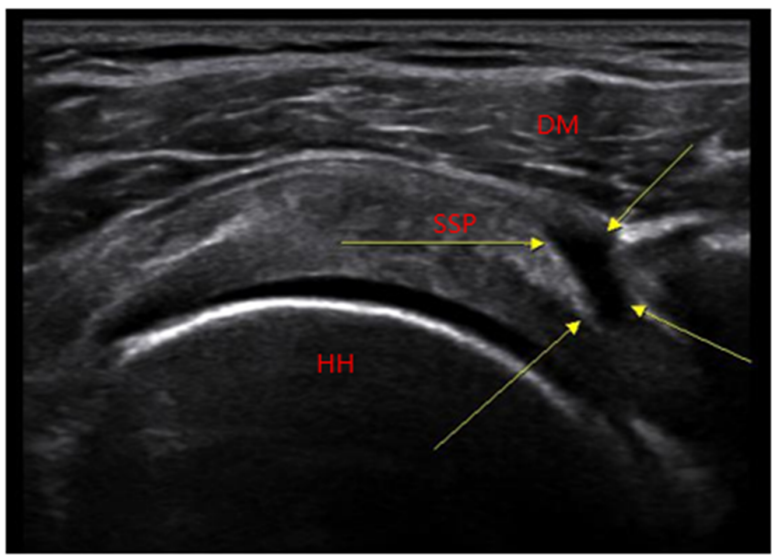

Figure 2. Ultrasound (US) image before the first treatment of Case 1.

JOAS: 43 points to 88 points.

NRS: 8 to 2 .

\section{8) Radiological findings}

The shoulder ultrasound was taken on Aug. 10 2021, at Samse Medical Hospital. The image showed widening of the acromioclavicular (AC) joint cavity and partial narrowing of the glenohumeral (GH) joint cavity (Fig. 2). The image showed the

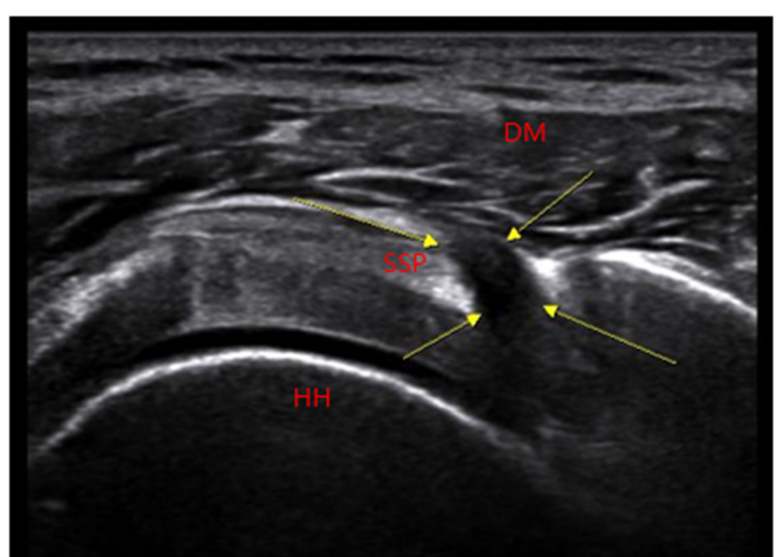

Figure 3. US image after 20 treatments of Case 1.

deltoid muscle (DM), the humeral head $(\mathrm{HH})$, and the supraspinatus tendon (SST).

Afterwards, an ultrasound was taken on Sep. 10 2021, and showed recovery of the partial tear on the proximal SST (30\%) (Fig. 3).

\section{Case 2}

1) Participant

Lee XX (Male, 63 years old)

2) Diagnoses

The diagnoses were as follows:

(1) Calcified tendinitis on SST.

(2) Partial adhesive gleno-humeral joint capsulitis.

\section{3) Chief complaints}

Left cervical pain radiating down to fingers, shoulder pain 
A.

10

8

NRS

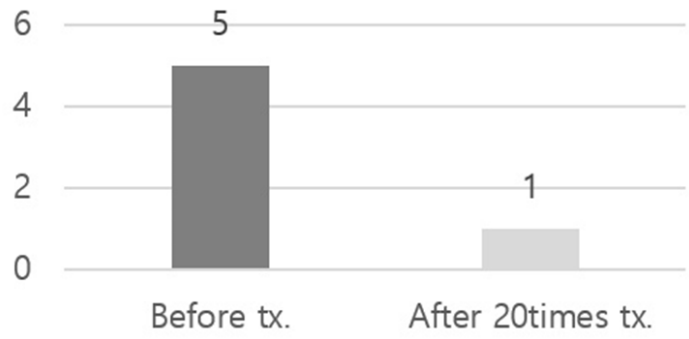

Figure 4. Assessment of pain in Case 2.

Table 3. JOAS of Case 2

\begin{tabular}{lcc}
\hline & Before tx. & After 20 times tx. \\
\hline Pain (30) & 20 & 25 \\
Function (20) & 11 & 20 \\
Range of motion (30) & 19 & 30 \\
Radiograph evaluation (5) & 3 & 3 \\
Joint stability (15) & 10 & 15 \\
Total (100) & 63 & 93 \\
\hline
\end{tabular}

on movement, limitation of movement, and night pain.

\section{4) Onset and cause}

Onset in Jun. 2021 with symptoms that worsened on Jul. 10, 2021.

\section{5) Present status}

Treatments of cervical traction, local injections at the orthopaedic clinic for two weeks.

\section{6) Treatment period}

The patient was treated between 14 Jul. 2021 and 30 Sep. 2021.

\section{7) Progress (Fig. 4, Table 3)}

Progress in the treatment of Case 2

Shoulder pain: 2021.07.14 (before treatment): 2021.09.30 (after 20 treatment)

JOAS: 63 versus 93

NRS: 5 versus 1
B.
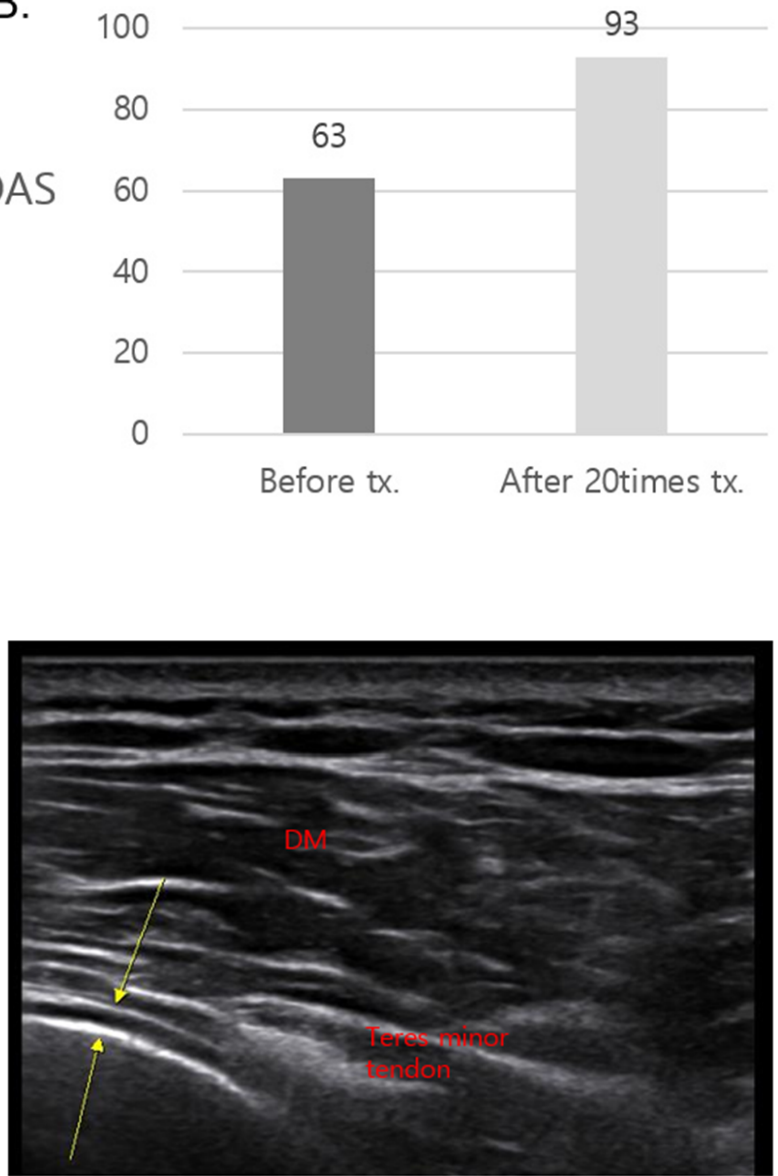

Figure 5. US image before the first treatment in Case 2.

\section{8) Radiological findings}

The ultrasound was taken on Aug 13 2021, and showed mild tendinopathy on the bicep tendon and subscapularis tendon (Fig. 5).

Afterwards, an ultrasound image was taken on Oct. 1, 2021 with no interval change shown (Fig. 6).

\section{DISCUSSION}

Shoulder pain is mostly derived from disorders of the rotator cuff which consists of supraspinatus, infraspinatus, teres minor, and subscapular tendon, though sometimes due to cervical nerve compression and referral of visceral diseases. Supraspinatus acts in conjunction with the other cuff muscles during $\mathrm{GH}$ movement to bring the head into the glenoid fossa, exerting minimal rotary action and the remaining cuff muscles, by their origin and insertion, have more of a downward pull and thus depress the head of humerus in a downward rotary direction 


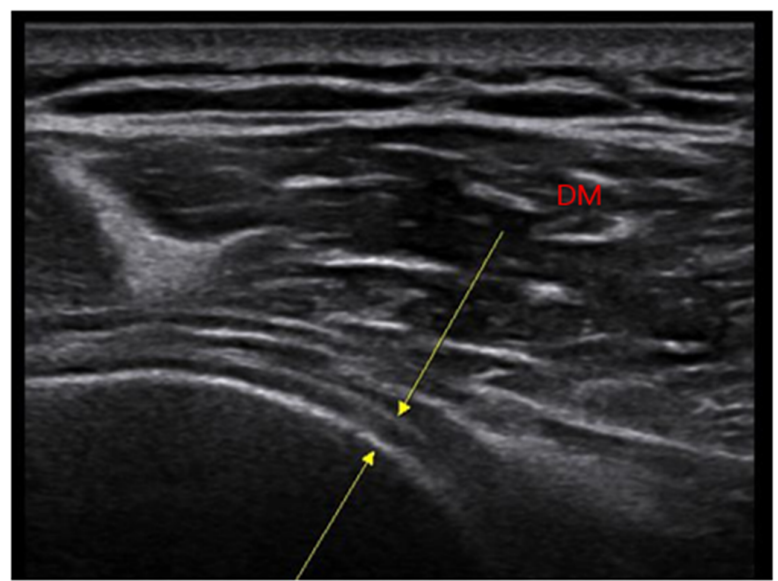

Figure 6. US image after 20 treatments in Case 2.

[19]. Rotator cuff problems lead to impingement tendonitis and adhesive capsulitis also known as frozen shoulder with a weakening of abduction, external rotation, and night pain. Frozen shoulder gets less painful depending on the stage over several months $[1,2]$.

Many Western conventional treatments for shoulder pain are used such as oral drug treatment, local injections, and nondrug physiotherapy treatment, but surgical decompression must be done if joint distension, sustaining symptoms, and continued functional disability follows after 6 months of physical therapy [4, 11, 20-23]. Jason et al. recommended acupuncture as a nonsurgical treatment though Kim et al. asserted that the efficacy of acupuncture on AC was not accurately assessed [11, 12]. However, Park et al. suggested that acupuncture may be an effective and safe treatment for impingement syndrome, and MacPherson claimed that the effect of acupuncture lasts over 12 months $[13,24]$. In order to effectively treat shoulder pain, many methods have been used such as electro-acupuncture, rehabilitation, trigger point dry needling with manual pressure technique, and conservative Korean Medicine treatments such as MA, PA, herbal drugs, moxibustion, and Chuna manipulation along with AK's manipulation [25-29].

OIT was developed in 1964 by Goodheart, the inventor of $\mathrm{AK}$, and was practiced in shoulder treatments. He postulated that the muscle weakness was due to a microavulsion of the tendon from the periosteum, and that heavy digital massage in the sites of origin/insertion of the weak muscle can strengthen it [5, 6]. OIT was stimulated mostly on insertion site of supraspinatus, which is the superior facet of greater tuberosity of humerus as well as on coracoid process (around LU1), which is the origins of the short head of biceps brachii and coracobrachialis as well as the insertion of pectoralis minor.

Its characteristics are that OIT stimulates origins/insertions of corresponding muscles different from muscle massage, which stimulates muscle belly or golgi tendon organs.

After taking into consideration the various treatments, MA and PA treatments were practiced following OIT with JOAS and NRS being assessed. The JOAS was applied to compare the pre- and post-treatment outcomes. Takagishi et al. regarded a JOAS of $>90,81-90,71-80$, and $<71$ to indicate excellent, good, fair, and poor outcome, respectively [30]. The score of the first patient was improved from 43 to 88 while that of the second patient from 63 to 93 . The NRS of the first patient changed from 8 to 2 and that of the second patient 5 to 1 .

The impingement of the rotator cuff is the result of anomalies or abnormalities in the coracoacromial arch and features of impingement may be identified on a routine radiograph, ultrasound, computerized tomography, and magnetic resonance imaging $[17,31]$. Case 1 showed changes in the ultrasound image from $40 / 50 \%$ to $30 \%$ in partial tear on SST, but case 2 showed no change in partial adhesive GH capsulitis. The reason for these ultrasound results must be pursued in the future.

\section{CONCLUSION}

The combined MA, PA, and OIT treatments were effective against shoulder pain such as impingement syndrome and partial adhesive capsulitis.

\section{CONFLICT OF INTEREST}

The authors have no conflicts of interest to declare.

\section{ORCID}

Chang-Beohm Ahn, https://orcid.org/0000-0003-2612-1495 Joon-Yong Noh, https://orcid.org/0000-0002-1377-7279 Hyun-Min Yoon, https://orcid.org/0000-0003-3645-6109 Cheol-Hong Kim, https://orcid.org/0000-0003-2058-0762 Ung-Kwan Song, https://orcid.org/0000-0001-6415-8606

\section{REFERENCES}

1. Murphy RJ, Carr AJ. Shoulder pain. BMJ Clin Evid. 2010;2010: 1107.

2. Japanese Orthopaedic Association. J JOA. 1987;61:623-9. 
3. Hawker GA, Mian S, Kendzerska T, French M. Measures of adult pain: Visual Analog Scale for Pain (VAS Pain), Numeric Rating Scale for Pain (NRS Pain), McGill Pain Questionnaire (MPQ), Short-Form McGill Pain Questionnaire (SF-MPQ), Chronic Pain Grade Scale (CPGS), Short Form-36 Bodily Pain Scale (SF36 BPS), and Measure of Intermittent and Constant Osteoarthritis Pain (ICOAP). Arthritis Care Res (Hoboken). 2011;63 Suppl 11:S240-52.

4. Bonutti PM, Hawkins RJ. Rotator cuff disorders. Baillieres Clin Rheumatol. 1989;3(3):535-50.

5. Walther DS. Applied kinesiology: synopsis. 2nd ed. Pueblo: Systems DC; 2000. p. 45-6.

6. Leaf D. Applied kinesiology flowchart manual. 3rd ed. Plymouth: David W. Leaf; 1995. 39 p.

7. Liu S, Zhang CS, Cai Y, Guo X, Zhang AL, Xue CC, et al. Acupuncture for post-stroke shoulder-hand syndrome: a systematic review and meta-analysis. Front Neurol. 2019;10:433.

8. Green S, Buchbinder R, Hetrick S. Acupuncture for shoulder pain. Cochrane Database Syst Rev. 2005;(2):CD005319.

9. Choi S, Kim KH. Acupuncture for symptomatic rotator cuff disease: protocol for a systematic review and meta-analysis. Medicine (Baltimore). 2020;99(2):e18716.

10. Ben-Arie E, Kao PY, Lee YC, Ho WC, Chou LW, Liu HP. The effectiveness of acupuncture in the treatment of frozen shoulder: a systematic review and meta-analysis. Evid Based Complement Alternat Med. 2020;2020:9790470.

11. Ramirez J. Adhesive capsulitis: diagnosis and management. Am Fam Physician. 2019;99(5):297-300.

12. Kim JE, Kim SP, Kim AR, Park HJ, Kwon O, Jung SY, et al. Acupuncture treatment of adhesive capsulitis of the shoulder: a randomized controlled pilot trial. J Acupunct Res. 2018;35(3):1208.

13. Park JE, Kim WY, Lee SJ, Oh DY, Lee MC, Jeon MK, et al. Acupuncture treatment for shoulder impingement syndrome: a review of randomized controlled trials. J Acupunct Res. 2021; 38(3):175-82.

14. Shen L, Lee JH, Joo JC, Park SJ, Song YS. Bee venom acupuncture for shoulder pain: a systematic review and meta-analysis of randomized controlled trials. J Pharmacopuncture. 2020;23(2): 44-53.

15. Lim SM, Lee SH. Effectiveness of bee venom acupuncture in alleviating post-stroke shoulder pain: a systematic review and meta-analysis. J Integr Med. 2015;13(4):241-7.

16. Reaves W, Bong C, Kelley D. The acupuncture handbook of sports injuries \& pain. Boulder: Hidden Needle Press; 2009. p. 275-302.

17. Backer M, Hammes MG. Acupuncture in the treatment of pain: an integrative approach. Edinburgh: Churchill Livingstone; 2010. p. 327-35.

18. Gellman H. Acupuncture treatment for musculoskeletal pain: a textbook for orthopaedics, anesthesia, and rehabilitation. London: CRC Press; 2002. p. 129-31.

19. Cailliet R. Shoulder pain. 2nd ed. Philadelphia: Davis Company; 1981. p. 20-1, 38-9.

20. Neviaser AS, Neviaser RJ. Adhesive capsulitis of the shoulder. J Am Acad Orthop Surg. 2011;19(9):536-42.

21. Revel M, Ghanem N. [Adhesive capsulitis of the shoulder]. Rev Prat. 1999;49 Suppl 13:1406-8. French.

22. Page MJ, Green S, McBain B, Surace SJ, Deitch J, Lyttle N, et al. Manual therapy and exercise for rotator cuff disease. Cochrane Database Syst Rev. 2016;2016(6):CD012224.

23. Favejee MM, Huisstede BM, Koes BW. Frozen shoulder: the effectiveness of conservative and surgical interventions--systematic review. Br J Sports Med. 2011;45(1):49-56.

24. MacPherson H, Vertosick EA, Foster NE, Lewith G, Linde K, Sherman KJ, et al. The persistence of the effects of acupuncture after a course of treatment: a meta-analysis of patients with chronic pain. Pain. 2017;158(5):784-93.

25. Lo MY, Wu CH, Luh JJ, Wang TG, Fu LC, Lin JG, et al. The effect of electroacupuncture merged with rehabilitation for frozen shoulder syndrome: a single-blind randomized sham-acupuncture controlled study. J Formos Med Assoc. 2020;119(1 Pt 1):81-8.

26. Hall ML, Mackie AC, Ribeiro DC. Effects of dry needling trigger point therapy in the shoulder region on patients with upper extremity pain and dysfunction: a systematic review with metaanalysis. Physiotherapy. 2018;104(2):167-77.

27. De Meulemeester KE, Castelein B, Coppieters I, Barbe T, Cools A, Cagnie B. Comparing trigger point dry needling and manual pressure technique for the management of myofascial neck/ shoulder pain: a randomized clinical trial. J Manipulative Physiol Ther. 2017;40(1):11-20.

28. Ahn CB, Lee SJ, Park YB, Park YL. Effect of combined traditional acupuncture and applied kinesiology on lumbar diseases. J Acupunct Res. 2020;37(2):94-101.

29. Lee SJ, Ahn CB. Effect of combined traditional acupuncture, pharmacopuncture and applied kinesiology on lumbar diseases of resident patients. J Pharmacopuncture. 2019;22(3):184-91.

30. Kawakami J, Gotoh M, Matsuura K, Shin K, Fujito I, Tanaka A, et al. Cut-off values of the Japanese Orthopaedic Association score corresponding to Constant scores for evaluating outcomes in rotator cuff tear. J Orthop Surg (Hong Kong). 2017;25(2): 2309499017720002.

31. Berquist TH. Musculoskeletal imaging companion. 2nd ed. Philadelphia: Lippincott Williams \& Wilkins; 2007. p. 462. 